and prioritised by hospital staff and the "little things" that are extremely important to these patients are not overlooked. Their need for routine, familiarity and sensory and play facilities was evident. Without their care and treatment being individualised to meet their very specific needs, CYP with LD can experience unnecessary emotional distress and physical harm.

\section{G197(P) NEUROIMAGING IN NEUROFIBROMATOSIS TYPE 1: OUTCOMES FROM A TARGETED APPROACH- EXPERIENCE OF A SINGLE TERTIARY CENTRE}

R Mithyantha, H Royden, J Acharya, Z Bassi. Community Paediatrics, Alderhey Children's Hospital NHS Tust, Liverpool, UK

\subsection{6/archdischild-2015-308599.191}

Aims Routine imaging is not recommended for patients with neurofibromatosis 1 (NF1) and a clinical approach is used to guide need for investigations. We aimed to determine clinical characteristics and symptoms which were most commonly associated with abnormalities on neuroimaging in children with NF1.

Methods We analysed 100 consecutive patients with an established diagnosis of NF1 seen in a tertiary NF1 clinic. Records of patients who had undergone imaging studies were reviewed to determine clinical presentation and associated comorbidities, along with neuroimaging results.

Results 59/100 children with NF1, (M:F = 55:45; median age 11.6 years (range 9 months-21 years) underwent cranial MRI scanning. The most common indications for MRI request being visual disturbance or abnormal fundoscopy (37\%), concerns regarding growth or puberty $(13.5 \%)$ and headaches $(10 \%)$. $91 \%$ had an abnormality on MRI, $66 \%$ of which were UBOs (unidentified bright objects). 13(22\%) optic pathway gliomas, 7 (11.8\%) non-glioma CNS tumours, $8(13.5 \%)$ had other CNS abnormalities. 10/59 (17\%) required surgical intervention. In 5/ $59(8 \%)$ children the scan was normal. Patients with learning difficulties and neuro-developmental disorders (such as ASD/ADD) had a significantly higher incidence of UBO (90\% vs. 40\%; p < 0.001). There was a higher incidence of non-UBO CNS lesions amongst the group with learning difficulties. 2 asymptomatic patients had incidental findings on scans done for other reasons; 1 of them required surgical intervention.

32/100 children had MRI spine, common indications being scoliosis (40\%) and back/neck pain (28\%). 34\% of these scans were abnormal. Chiari malformation, cervical syrinx and dural ectasia were the most common abnormalities. 5/27 (18\%) needed scoliosis surgery.

Conclusion Targeted imaging in our cohort identified a significant proportion of abnormalities. Children with NF1 and associated neurodevelopmental conditions (learning difficulties, ASD, ADHD) were more likely to have an abnormality on the scans, thus warranting a lower threshold for neuroimaging. For the efficient use of resources, a high index of clinical suspicion is essential for early identification of NF1 related complications.

\section{G198(P) CHILD AND ADOLESCENT PSYCHIATRY TRAINING DURING NEURODISABILITY GRID: AN EXPERIENCE BY THE TRAINEES IN THE UK}

V Mundada. Paediatric Neuroscience, Great Ormond Street Hospital, London, UK

10.1136/archdischild-2015-308599.192
Aim The neurology and neurodisability grid trainees should have a child and adolescent psychiatry experience equivalent to three months full time during their grid programme. The aim of our survey was to gather information and analyse the child and adolescent psychiatry training experience by the current paediatric neurodisability trainees.

Method This was a questionnaire based survey which was sent out to the existing neurodisability grid trainees in the UK via email. Total 10 questions were asked related to the child and adolescent psychiatry training experience.

Results Out of total 26 trainees to whom the survey was sent out, 16 responded (61.5\%). 60\% were working as ST8. The neurodisability grid programme was of two years for $60 \%$ of the trainees. Most of them (73\%) had already started their neuropsychiatry placement. Only 6\% found easy but 26\% thought that it was very difficult to access the training. $15 \%$ had to have a separate honorary contract with the child and family mental health (CAMHS) department. None was on a separate 3 month contract. In terms of satisfaction, one third seemed dissatisfied for various reasons and only $6 \%$ were very satisfied.

Some of the comments were- "CAMHS team members are reluctant to have the neurodisability trainee in the clinic unless it was a pre-school child with possible developmental problems"; "No learning disability CAMHS consultant in the service"; "CAMHS services were not sure of the competencies needed to be achieved"; "Difficult to get the placement and the service was quite stretched"; "Spent a lot of time chasing sessions"; "CAMHS consultants should be involved while submitting the grid posts to RCPCH"

Conclusion Child and adolescent mental health services (CAMHS) are lacking in the expertise and resources required to provide comprehensive assessments and ongoing management for those with developmental disabilities. Our survey highlights the need for the CAMHS service to engage the neurology and neurodisability grid trainees by understanding the competencies they need to achieve in child and adolescent psychiatry. Equally, the respective CSACs could also work in partnership with the child mental health CSAC to design a smooth and well-structured programme for these trainees.

\section{G199(P) AUDIT OF INFORMATION PROVIDED TO PARENTS OF CHILDREN WITH EPILEPSY}

MG Ghazavi. Paediatrics, South Tyneside NHS Foundation Trust, South Shields, UK

\subsection{6/archdischild-2015-308599.193}

Introduction Epilepsy is common in children. Diagnosis may impact negatively on the family. Witnessing a seizure can be very frightening. A wide range of emotions and reactions from the parents are reported once the diagnosis is made. High quality information for the family provided on time can be very beneficial. Involving the child and family in decision making is associated with good outcome and improve compliance.

Aim There are risks associated with epilepsy. Many professionals pay attention to medical management (medication) once a diagnosis is made. However, needs of the child and family for high quality information is ignored. NICE guideline has highlighted what information needs to be provided to the family and what safety measures to be taken. As with no epilepsy clinic locally, I performed an audit to look into our practice and to check it against the standards set by the NICE and National service 
framework for long term conditions in order to improve the service delivery to children with epilepsy.

Method A questionnaire was developed using CG137epilepsy NICE guideline. Patients with epilepsy who were seen over the period of January-May 2014 were selected to receive the questionnaires that included parents feeling once the diagnosis is made. Patients were seen both in hospital and community settings.

Results 40 received the questionnaires, 34 returned (85\%). Most (30/34) were younger than 10 years at the time of diagnosis. Co-morbidities were seen in $62 \%$ including cerebral palsy, learning difficulty, autism, ADHD and some had combinations. The high number relates to seeing more children in special schools over the period of the audit. 18/40 reported significant emotional problems at diagnosis: sad, terrified, devastated and shocked. Information on lifestyle including water safety was provided to $25 / 34$ (70\%). Information on SUDEP was not provided to $23 / 34(68 \%)$.

Conclusion There is a wide variation in practice within the Department. There is no epilepsy clinic with no epilepsy nurse. The information provided to patients was very patchy, too little or given too late.

Action Proforma was put in place, covered classification as per Multi-axial diagnostic scheme: Description of seizures, Seizure type, Syndrome, Aetiology and Co-morbidity. Referral letter is sent to the community nursing team for early home visit and an early liaison with school to improve outcome. Group training for the parents was commenced.

\section{G200(P) A CASE HIGHLIGHTING THE IMPACT OF UNCORRECTED SCOLIOSIS}

S Sanapala. Southmead Hospital, North Bristol NHS Trust, Bristol, UK

10.1136/archdischild-2015-308599.194

Method This is a case of a previously well 17 year old girl with cerebral palsy secondary to Lissencephaly. She presented with a history of intractable vomiting progressing over the course of a few months secondary to severe worsening scoliosis.

Upon further investigation, it was felt that the extensive degree of her scoliosis was rotating her abdominal anatomy in such a manner to be the main cause of her vomiting. She was reviewed by the orthopaedic team who felt that correction of her scoliosis was indicated however this was not without significant risks to the patient especially given her extensive recent weight loss secondary to the vomiting. However, without the procedure the patient would continue to vomit and losing weight and may eventually die.

Result The parents of the patient were keen to proceed with the operation despite the high risks as they felt their daughter's quality of life had deteriorated significantly since the onset of the scoliosis and recurrent vomiting.

The patient underwent the scoliosis correction and made a good recovery.

Conclusion This case presents a dilemma in which without correction of her scoliosis, the patient would continue to vomit and lose weight (medical therapy had failed). However, undertaking such a major operation in this patient carried a significant mortality risk.

The case also highlights the importance of close follow up of these patients as this child did not develop scoliosis until her teenage years and she missed her follow up appointments due to an address change.

Pictures of the scoliosis (X ray/CT are available to accompany the case)

\section{G201(P) DO BASELINE BLOOD PRESSURE AND HEART RATE IN CHILDREN WITH A SPINAL CORD INJURY VARY DEPENDING ON THEIR LEVEL OF INJURY?}

${ }^{1} \mathrm{E}$ Gustafsson Oberink, 'S Gale, ${ }^{2} \mathrm{~A}$ Graham. National Spinal Injury Unit, Stoke Mandeville Hospital, Aylesbury, UK

\subsection{6/archdischild-2015-308599.195}

Children with a spinal cord injury may have blood pressure and heart rate differences from the expected values of that age. Understanding this potential problem is important for optimal management.

Aims To establish any association between level of spinal injury and ASIA (American Spinal Injury Association) impairment scale severity score with heart rate, blood pressure and diurnal variation in blood pressure. The association between age, gender and BMI on heart rate and blood pressure was also explored.

Method Retrospective records were reviewed for 32 children admitted to a National Spinal Injury Centre for more than 4 rehabilitation periods between 2011 and 2013 (107 admissions). Patient and injury data were established. The first recorded morning and evening blood pressures and heart rates were collated.

Data was analysed using MS Excel 2010 and IBM SPSS v.20. Results The mean age at first admission was 10.2 years, $41 \%$ were male, and $59 \%$ had an injury at or above T6. Only $12 \%$ of the admissions had complete cardiovascular data.

The associations between ASIA score and BP centile group; ASIA score and diurnal variation; level of injury and BP centile group; and level of injury and diurnal variation, were not statistically significant.

Increasing age was significantly associated with a lower heart rate (OR 0.094, p value <0.001) and systolic BP with increasing BMI (OR 2.97, 95\% CI(1.439, 6.137)

Conclusion The changes observed with age and BMI can be accepted as normal physiological change. That no statistical association between the injury related factors, ASIA score and level of injury, and cardiovascular measures was observed could be due to the poor data quality, and no conclusion can therefore be reached from this finding.

Improved measurement and recording of height, weight and cardiovascular observations is paramount for optimal cardiovascular management in this patient group.

\section{G202(P) SLEEP MANAGEMENT IN AUTISTIC SPECTRUM DISORDER}

${ }^{1} S$ Jmor, ${ }^{2} E$ Abbas. 'Medical School, Birmingham University, Birmingham, UK; ${ }^{2}$ Community Child Health, Wrightington Wigan and Leigh NHS FT, Wigan, UK

\subsection{6/archdischild-2015-308599.196}

Abnormal sleep affects 44-89\% of children with ASD. Currently there are no official UK guidelines for the management of sleep with ASD.

Aim To discuss sleep management using a case study of 9 year old boy with ASD. 